\title{
Cobalt Silicide Nanostructures: Synthesis, Electron Transport, and Field Emission Properties
}

Chun-I Tsai, ${ }^{\dagger, \#}$ Ping-Hung Yeh, ${ }^{\dagger, *, \#}$ Chiu-Yen Wang, ${ }^{\dagger}$ Han-Wei Wu, ${ }^{\dagger}$ Uei-Shin Chen, ${ }^{\dagger}$ Ming-Yen Lu, ${ }^{\dagger}$ Wen-Wei Wu, ${ }^{,}$Lih-Juann Chen, ${ }^{*}, \dagger$ and Zhong-Lin Wang*,*

${ }^{\dagger}$ Department of Materials Science and Engineering, National Tsing Hua University, Hsinchu 300, Taiwan, Republic of China, ${ }^{*}$ School of Materials Science and Engineering, Georgia Institute of Technology, Atlanta, Georgia 30332, and ${ }^{\S}$ Department of Materials Science and Engineering, National Chiao Tung University, Hsinchu 300, Taiwan, Republic of China. ${ }^{\#}$ These authors contributed equally to this work.

Received May 17, 2009; Revised Manuscript Received August 15, 2009

\begin{abstract}
Cobalt silicide nanostructures have been synthesized by a spontaneous chemical vapor transport and reaction method. The temperature and the vapor flow rate were shown to critically influence the growth of nanostructures. The effects of two main parameters on the growth of nanostructures were discussed. The phases formed were determined by the Gibbs free energy changes in the reactions. Various phases $\left(\mathrm{CoSi}, \mathrm{Co}_{2} \mathrm{Si}\right)$ and morphologies, such as single-stem nanowires, threedimensional (3D) nanowire networks, and aloelike nanostructures, have been synthesized. Very low turn-on field $(1.42 \mathrm{~V} / \mu \mathrm{m})$ and good conductance obtained from field-emission and electrical property measurements, respectively, indicate that CoSi nanowires are potentially useful for electronic devices.
\end{abstract}

\section{Introduction}

One-dimensional (1D) nanostructures such as nanowires (NWs), nanobelts, nanotubes, and nanorods, are attracting much interest because of their unique physical properties. ${ }^{1-3}$ These nanostructures are expected to play an important role as interconnects and functional units in fabricating electronic, optoelectronic, and electromechanical devices. ${ }^{4-9}$ Metal silicides constitute an important group of compounds that are used in silicon-based devices. ${ }^{10-12}$ Cobalt silicide is known to be a high-melting point material with very low resistivity, which offers the possibility of electrical interconnections in nanoscale. ${ }^{13}$ Cobalt silicides have been shown to be promising cold cathode materials for emission due to the high thermal and chemical stability. ${ }^{14}$ A number of metal silicide nanowires, including $\mathrm{TaSi}_{2}, \mathrm{TiSi}_{2}$, and $\mathrm{Ni}$ silicides nanowires, were also reported to possess excellent field-emission properties. ${ }^{15-19}$

Various effective strategies have been developed for the synthesis of silicide nanowires. ${ }^{13,20-22} \mathrm{TiSi}_{2}$ nanowires were grown in a single direction on silicon surface by reactive deposition epitaxy. ${ }^{21} \mathrm{CoSi}$ and $\mathrm{FeSi}$ NWs were synthesized via chemical vapor deposition (CVD) of single-source precursor and serve as building block of magnetic semiconducting $\mathrm{Fe}_{1-x} \mathrm{Co}_{x} \mathrm{Si}$ NWs suitable for silicon-based spintronics. ${ }^{22-24}$ $\mathrm{TaSi}_{2}$ nanowires have been synthesized on a Si substrate by annealing $\mathrm{NiSi}_{2}$ films in an ambient containing Ta vapor. ${ }^{15}$ Single-crystalline metal silicide nanowires synthesized by metal halide precursors were reported recently. ${ }^{25-30}$

In this paper, we report the growth of $\mathrm{Co}_{2} \mathrm{Si}$ and $\mathrm{CoSi}$ nanostructures by a spontaneous chemical vapor transport growth method. Both reaction temperature and vapor flow rate were found to critically influence the growth of nanostructures in the present study. In addition, a variety of

*To whom correspondence should be addressed. E-mail: ljchen@, mx.nthu.edu.tw (L.-J.C.); zlwang@gatch.edu (Z.-L.W.). Tel.: 886-35731166 Fax: 886-3-5718328. structures and morphologies of cobalt silicide nanostructures, such as single-stem $\mathrm{CoSi}$ and $\mathrm{Co}_{2} \mathrm{Si}$ nanowires, 3D CoSi nanowire networks, and aloelike $\mathrm{Co}_{2} \mathrm{Si}$ nanostructures, were grown under suitable conditions. The field emission properties of various structures and morphology of CoSi nanostructures were compared. The growth of $\mathrm{Co}_{2} \mathrm{Si}$ and $\mathrm{CoSi}$ nanowires by similar synthesis methods was previously reported. ${ }^{26,31}$

\section{Experimental Section}

Single crystal Si (001) wafers were used as substrates. Prior to the loading into the two-zone furnace at the atmosphere pressure with an argon (Ar) flow, the wafer was cleaned with 1\%-buffered HF. The samples were placed at the downstream and the cobalt chloride powder at the upstream. The powders were heated at $600{ }^{\circ} \mathrm{C}$ and the heating zone was set at temperatures of $800-1000{ }^{\circ} \mathrm{C}$ downstream. The two heating centers were at a distance of $20 \mathrm{~cm}$ apart. The exact temperature of the substrate was measured with a thermocouple attached to the substrate. The temperature was ramped up from room temperature to reaction temperature in $60 \mathrm{~min}$. The precursor vapors were carried by the Ar flow, which was set at 50, 150, and 300 sccm, and reacted with silicon substrates placed downstream for $2 \mathrm{~h}$ unless otherwise mentioned. The furnace was then allowed to cool to room temperature. The phase formation was investigated by X-ray diffraction (XRD). The morphology of products was examined with a field emission scanning electron microscope (SEM) (JSM-6500F). The phases and compositions of nanostructures were obtained with a JEOL-2010 transmission electron microscope (TEM), equipped with an energy-dispersive spectrometer (EDS). The field emission characteristics were measured with a Keithley 237 tester.

\section{Results and Discussion}

In a previous work, the pathways for $\mathrm{CoCl}_{2}$ vapor to react with $\mathrm{Si}$ substrate to form $\mathrm{CoSi}$ nanowires were proposed as follows. ${ }^{26}$

$$
\begin{aligned}
& 2 \mathrm{CoCl}_{2}(\mathrm{~g})+3 \mathrm{Si}(\mathrm{s}) \leftrightarrow 2 \mathrm{CoSi}(\mathrm{s})+\mathrm{SiCl}_{4}(\mathrm{~g}) \\
& \mathrm{CoCl}_{2}(\mathrm{~g})+\mathrm{SiCl}_{4}(\mathrm{~g}) \leftrightarrow \mathrm{CoSi}(\mathrm{s})+3 \mathrm{Cl}_{2}(\mathrm{~g})
\end{aligned}
$$




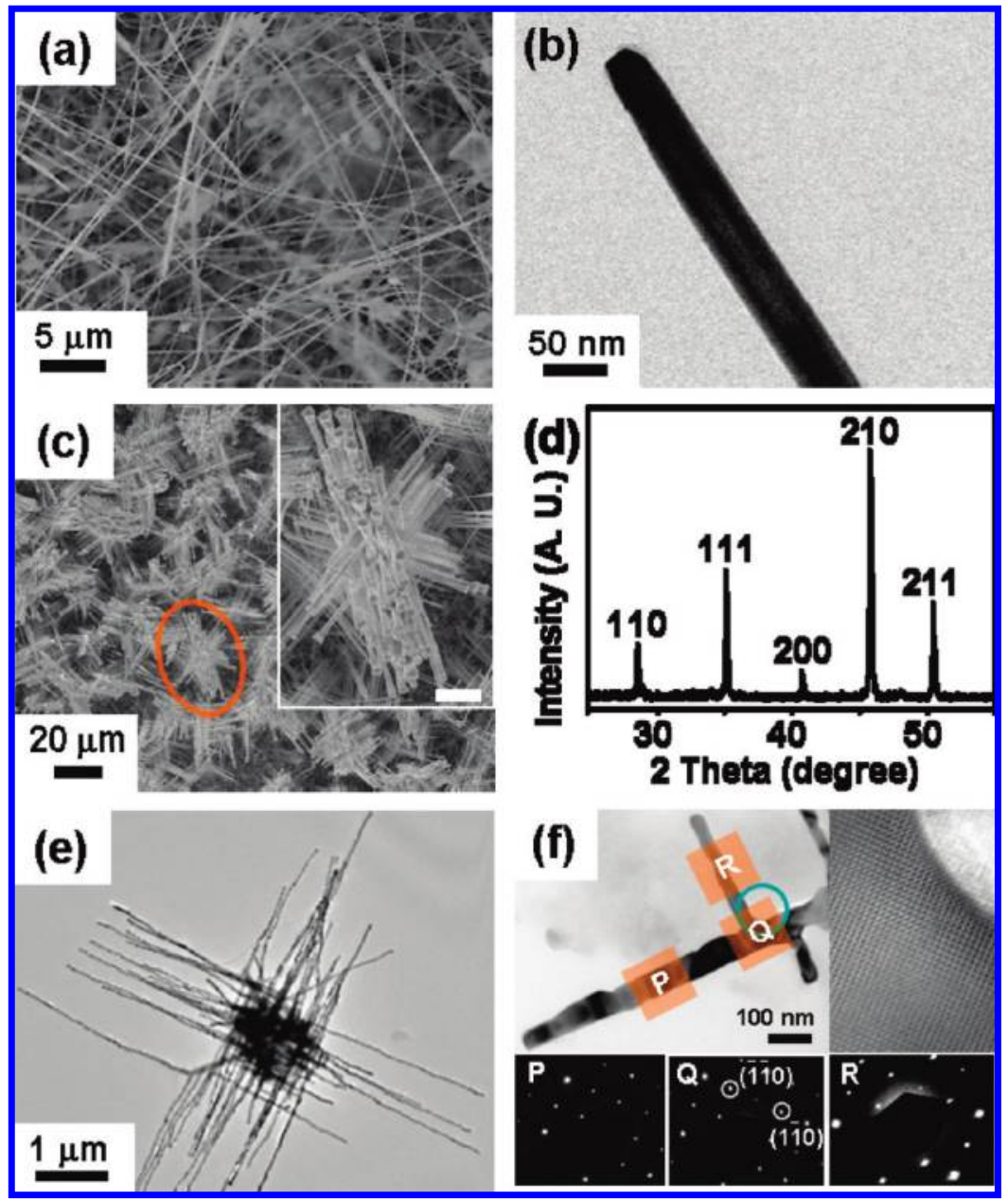

Figure 1. (a) Representative SEM image of CoSi nanowires grown at $750{ }^{\circ} \mathrm{C}$ in region $\mathrm{A}$. (b) TEM image of a CoSi nanowire grown at $750{ }^{\circ} \mathrm{C}$ in region A. (c) Low-magnification SEM images of the CoSi nanowire networks grown at $780^{\circ} \mathrm{C}$ in region A. Inset: High-magnification SEM image. The scale bar is $5 \mu \mathrm{m}$. (d) XRD pattern from a sample of CoSi nanowire networks grown at $780{ }^{\circ} \mathrm{C}$ in region A. TEM images (e) showing a complete 3D nanowire network and (f) a broken nanowire network segment grown at $780{ }^{\circ} \mathrm{C}$ in region A. Insets are the SAEDs from the regions $\mathrm{P}, \mathrm{Q}$, and $\mathrm{R}$ as well as HRTEM image of an intersection.

The reactions for the formation of $\mathrm{Co}_{2} \mathrm{Si}$ nanowires are likely to follow similar pathways.

$$
\begin{gathered}
2 \mathrm{CoCl}_{2}(\mathrm{~g})+2 \mathrm{Si}(\mathrm{s}) \leftrightarrow \mathrm{Co}_{2} \mathrm{Si}(\mathrm{s})+\mathrm{SiCl}_{4}(\mathrm{~g}) \\
2 \mathrm{CoCl}_{2}(\mathrm{~g})+\mathrm{SiCl}_{4}(\mathrm{~g}) \leftrightarrow \mathrm{Co}_{2} \mathrm{Si}(\mathrm{s})+4 \mathrm{Cl}_{2}(\mathrm{~g})
\end{gathered}
$$

The growth processes of cobalt silicide $\left(\mathrm{CoSi}, \mathrm{Co}_{2} \mathrm{Si}\right)$ nanowires include two steps. First, the silicide particles were formed on the substrate. Second, self-catalyzed nanowires were grown from the silicide particles. ${ }^{25}$ (see the Supporting Information, S1) By controlling the vapor pressure of the precursor and the temperature of the substrate, we can obtain a variety of structures and morphologies of the cobalt silicide $\left(\mathrm{CoSi}, \mathrm{Co}_{2} \mathrm{Si}\right)$ nanostructures. The morphologies are quite different at various substrate temperatures. At $610{ }^{\circ} \mathrm{C}$, only particles and very short nanowires were observed. At $790^{\circ} \mathrm{C}$, because of the fast growth rate, the micrometer-sized particles were formed. A high density of single-stem $\mathrm{CoSi}$ nanowires was formed at $750{ }^{\circ} \mathrm{C}$, as shown in Figure 1a. The diameters and lengths of the synthesized nanowires are $40-80 \mathrm{~nm}$ and tens of micrometer, respectively. Compared with nanowires formed at $790{ }^{\circ} \mathrm{C}$ with a diameter of about $100 \mathrm{~nm}$, moderate reaction temperature is more conducive to the nucleation and growth of nanowires.
By adjusting the carrier gas flow rate, similar reaction products were obtained at the three temperatures with different Ar flow rates. In particular, the diameter of nanowires was not affected significantly by the Ar flow rate. The variation in Ar flow rates only affects the densities and lengths of nanowires. For example, decreasing Ar flow rate from 300 to $50 \mathrm{sccm}$ led to the lower density and shorter nanowires. By controlling the two main parameters, the Ar flow rate and the temperature of substrate, the various morphologies of cobalt silicide $\left(\mathrm{CoSi}, \mathrm{CO}_{2} \mathrm{Si}\right)$ nanostructures can be obtained. The morphologies of the nanostructures and the diameter of nanowires were influenced by reaction temperature. The densities and lengths of nanowires were affected by Ar flow rate. The nanostructures formed at various temperature and flow rate are shown in the Supporting Information, S2.

The optimum synthesis condition for single-stem $\mathrm{CoSi}$ nanowires was realized with the heating zone set at $900{ }^{\circ} \mathrm{C}$ and $\mathrm{Ar}$ flow rate maintained at $300 \mathrm{sccm}$. A string of $\mathrm{Si}$ substrates was placed horizontally in the furnace. The temperatures of three regions in the heating zone were measured with a thermocouple attached to the samples. Apparently, the nanostructures of cobalt silicides $\left(\mathrm{CoSi}, \mathrm{Co}_{2} \mathrm{Si}\right)$ were influenced by a slight variation in vapor flux with the location in the furnace. The nanostructures formed at different regions 


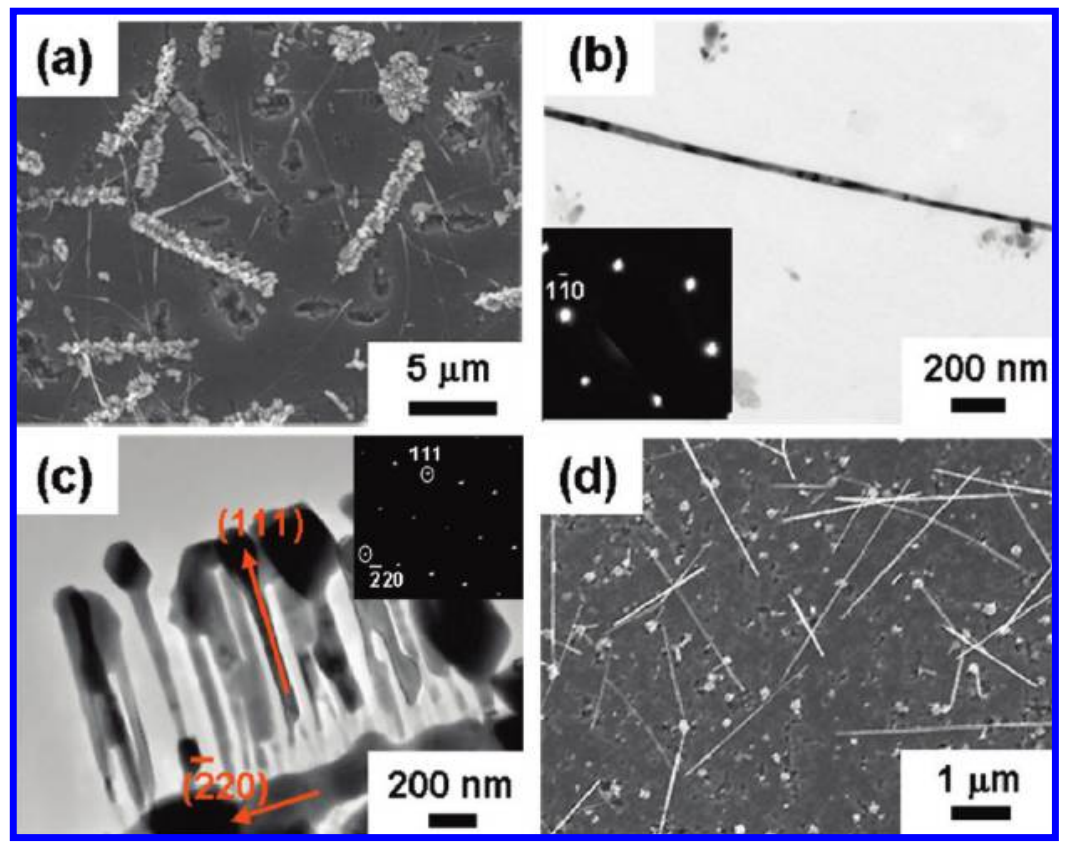

Figure 2. (a) SEM image of $\mathrm{Co}_{2} \mathrm{Si}$ nanowires formed in region B. TEM images of (b) a single-stem nanowire and (c) aloelike nanostructures. (d) Representative SEM image of straggled CoSi nanowires grown in region C.

in the furnace with the heating zone set at $900{ }^{\circ} \mathrm{C}$ and the $\mathrm{Ar}$ flow rate maintained at $300 \mathrm{sccm}$ are depicted in the Supporting Information, S3. Region A is the closest among the three regions to the source boat, and the vapor flux is expected to be the highest. The region at the heating center with a decreased vapor flux and the highest temperature is referred to as region B. Region C is far away from the source boat with the lower vapor flux; the temperature is close to that of $\mathrm{A}$.

The samples in region A near the source boat encountered the highest vapor pressure at a temperature range of 750$830^{\circ} \mathrm{C}$. The TEM image of a single-stem nanowire grown at $750^{\circ} \mathrm{C}$ in region $\mathrm{A}$ is illustrated in Figure $1 \mathrm{~b}$. The diffraction spots in the SAED pattern (not shown) can be ascribed to the cubic B20 type CoSi. The CoSi nanowire networks were synthesized at the particular zone with a higher temperature $\left(780^{\circ} \mathrm{C}\right)$ than that for single-stem nanowire growth. The high yield of 3D nanowire networks can be observed from the lowmagnification SEM image and the morphology of 3D nanowire networks can be clearly demonstrated in a high-magnification image, as shown in Figure 1c. The size of each network is about $10-30 \mu \mathrm{m}$. The constituents of $3 \mathrm{D}$ network structure are nanowires with diameters around $100 \mathrm{~nm}$ in width and they intercross with each other to form the 3D networks. The peaks of the XRD from the 3D nanowire networks grown at $780^{\circ} \mathrm{C}$ in region A shown in Figure 1d can all be ascribed to the CoSi phase. The TEM images of a complete 3D nanowire network and a broken nanowire network segment are shown in panels e and $\mathrm{f}$ in Figure 1, respectively. The angle of the junction is nearly equal to $90^{\circ}$. The SAED patterns were obtained from regions $\mathrm{P}, \mathrm{Q}$, and $\mathrm{R}$, indicating that the entire network is single-crystalline CoSi. The nanowires $\mathrm{P}$ and $\mathrm{R}$ are grown along the [110] and [110] directions, respectively, and rotate by $90^{\circ}$ with each other. HRTEM image of the intersections of the network further revealed that no dislocations were present to influence the branching growth, as shown in the inset of Figure 1f. In a previous work, branched morphology of nanowires was correlated with the high supersaturation ratio. ${ }^{25}$ Accordingly, as the Ar flow rate was decreased from 300 to $50 \mathrm{sccm}, 3 \mathrm{D}$ nanowire networks were no longer grown.

The substrate in region $\mathrm{B}$ was positioned at the comparatively high temperature zone $\left(880-900{ }^{\circ} \mathrm{C}\right)$. Two kinds of nanostructures were produced in the same area: single-stem nanowires and aloelike nanostructures. The diameter and length of the single-stem nanowires are 50-100 $\mathrm{nm}$ and several micrometers, respectively, as illustrated in Figure 2a. A TEM image and a SAED of single-stem nanowire are shown in Figure $2 b$. The SAED pattern reveals that the nanowire is single-crystalline and could be ascribed to the orthorhombic $\mathrm{Co}_{2} \mathrm{Si}$ structure. The size of each aloelike nanostructures and the diameter of its branch are about $10 \mu \mathrm{m}$ and $80-120 \mathrm{~nm}$, respectively. The TEM image and a SAED pattern of aloelike nanostructures are shown in Figure 2c. The SAED patterns obtained for the trunk, the head and the branch of the aloelike nanostructures can also be ascribed to orthorhombic $\mathrm{Co}_{2} \mathrm{Si}$ structure. The branches and trunk of the aloelike nanowires were found to grow along [111] and [220] directions, respectively. Notably, the discontinuous singlecrystalline structure at the intersection was replaced by an amorphous layer, which suggests that amorphous silicon oxide was formed at the local regions. The results differ from the branch structure obtained at region $\mathrm{A}$. The region $\mathrm{B}$ where aloelike nanostructures formed is located in the highest temperature zone with a diminished vapor flux compared to that of region A. The trunks of aloelike nanostructures formed first and the branches evolved from the trunk with the vapor achieving the sufficient degree of supersaturation to facilitate the growth. As the branches growth continued, amorphous silicon oxide was formed at the roots of the branches with the decreased supply of $\mathrm{CoCl}_{2}$ vapor. Moreover, as the vapor pressure was decreased with the diminished supply of the source vapor, the branched nanowires became trimmed and appeared to be terminated with spherical end to form aloelike nanostructures. A similar morphology of nanowires with a large head was previously observed for the TiSi nanopins. The varied size of the nanopins was also explained 
in terms of the diminishing source flux with time. ${ }^{32}$ The amorphous silicon oxide seen in Figure 2 is related to residual oxygen in the processing chamber. Cobalt silicide $(\mathrm{CoSi}$, $\mathrm{Co}_{2} \mathrm{Si}$ ) nanostructures were synthesized in the diffusion furnace with flowing Ar. It was therefore inevitable that residual oxygen was involved in the reactions.

The Gibbs free energies of the substances are shown in the Supporting Information, S4. The Gibbs free energies of the reaction 1 at the temperatures of $750^{\circ} \mathrm{C}$ (region $\mathrm{A}$ ) and $880^{\circ} \mathrm{C}$ (region $\mathrm{B}$ ) are $-1166 \mathrm{~kJ} / \mathrm{mol}$ and $-1158 \mathrm{~kJ} / \mathrm{mol}$, respectively. The Gibbs free energies of the reaction 2 at the temperatures of $750{ }^{\circ} \mathrm{C}$ (region A) and $880^{\circ} \mathrm{C}$ (region B) are $-1125 \mathrm{~kJ} / \mathrm{mol}$ and $-1255 \mathrm{~kJ} / \mathrm{mol}$, respectively. Consequently, reaction 1 , which has the lower Gibbs free energy at a temperature at $750{ }^{\circ} \mathrm{C}$, is the main reaction to lead to the formation of CoSi nanowires. On the other hand, $\mathrm{Co}_{2} \mathrm{Si}$ nanowires were produced at $880^{\circ} \mathrm{C}$ following the reaction 2 . The phases formed were determined by the Gibbs free energy changes in the reactions.

Region $\mathrm{C}$ was far away from the source boat and the temperature was close to that of region A. With lower vapor pressure, straggled single-stem nanowires were formed as revealed by the SEM image. No particles and etching marks on the flat substrate surface were observed. However, the density of the smooth and straight nanowires of 40-80 nm in diameter and $4-6 \mu \mathrm{m}$ in length was rather low, as illustrated in Figure 2d. The diameter of nanowires is identical to that in region $\mathrm{A}$ but somewhat shorter. The nanowires in region $\mathrm{C}$ are also of $\mathrm{CoSi}$ phase. $\mathrm{Co}_{2} \mathrm{Si}$ nanowires were synthesized only in the region $\mathrm{B}$. XRD spectrum (not shown) of products obtained from region A showed that all peaks can be ascribed to cubic structure CoSi. The result indicated that only CoSi nanowires were formed on $\mathrm{Si}$ substrate. No XRD data were obtained from the products in the region $\mathrm{C}$ because the density of straggled nanowires is very low. On the other hand, TEM analyses, including selected area electron diffraction pattern, high-resolution TEM, and energy-dispersive spectrum, of several nanowires obtained in the region $\mathrm{C}$ all indicated that the nanowires are single-crystalline CoSi.

Field-emission measurements were carried out in a vacuum chamber pumped to $\sim 1.0 \times 10^{-5}$ torr in pressure at room temperature and with an anode to sample distance of $180 \mu \mathrm{m}$. The turn-on field and threshold field are defined to be the fields required to produce a current density of 10 and $100 \mu \mathrm{A} /$ $\mathrm{cm}^{2}$, respectively. The three types of CoSi nanostructure are the high density single-stem nanowires, the 3D nanowire networks, and the straggled single-stem nanowires. The field emission data were taken from samples of $1 \mathrm{~cm}^{2}$ in size and represented the average effects from different nanostructures. For 3D nanowire networks, the distances between the NW emission tips to the anode were less than $180 \mu \mathrm{m}$. For the same samples, the measurements were made repeatedly. Stable and reproducible $J-E$ characteristics were only obtained for single-stem CoSi nanowires. The emission current density, $J$, as a function of applied field, $E$, is depicted in Figure 3. The turn-on fields of the high-density single-stem nanowires and the $3 \mathrm{D}$ nanowire networks are 1.42 and $2.20 \mathrm{~V} / \mu \mathrm{m}$. The threshold field of the high density single-stem nanowires is $2.05 \mathrm{eV}$. The values are much lower than the reported values of $\mathrm{TaSi}_{2}, \mathrm{TiSi}_{2}$, and Ni silicides nanowires. ${ }^{15-19}$ The turn-on field of the straggled single-stem nanowires was not stable and the best profile is shown in Figure 3. Compared to the 3D network structure, the turn-on field is lower and the emission current is higher for the single-stem nanowires. The Fowler-Nordheim $(\mathrm{FN})$ plot of $\ln \left(J / E^{2}\right)-1 / E$ of the single-stem CoSi nanowires

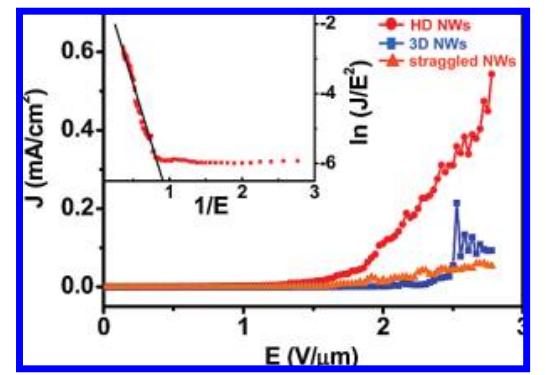

Figure 3. Field emission curves of the three type nanostructures: high density single-stem nanowires (HD NWs), the 3D nanowires networks, and the straggled single-stem nanowires. Inset: the FN plot of high density single-stem nanowires.

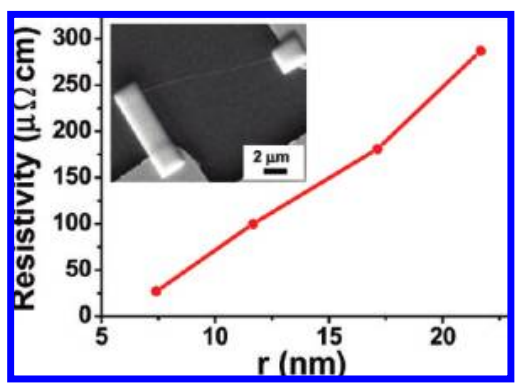

Figure 4. The curve depicting resistivity versus diameter of nanowires. Insets: the SEM image of the measurement structure.

is shown in the inset of Figure 3. By determining the slope of the FN plot, the field enhancement factor $\beta$ of single-stem nanowires was calculated to be $1 \times 10^{3}$, which is higher than other silicide nanowire emitters mentioned above. ${ }^{15-19}$ The field emission of single-stem nanowires is more efficient, homogeneous, and stable, as illustrated in Figure 3. The 3D network structure appeared to be less efficient than singlestem nanowires in current emission. For the 3D network, the sharp tips for emission are relatively scarce. Furthermore, the distribution of the electric charge in the 3D network structure may impede the emission of current at the tips as shown in Figure 3. It is worthwhile to mention that CoSi nanowires were sheathed with 3-5 nm silicon oxide. Dilute HF was used to remove the amorphous silicon oxide. However, the oxide regrew rapidly in air ambient. Therefore, the measurements of field emission properties were made for CoSi nanowires with silicon oxide sheath. The oxide coatings are expected to influence the field emission properties of nanowires. Measurement of field emission properties of $\mathrm{CO}_{2} \mathrm{Si}$ nanowires was attempted. However, the density of nanowires was so low that no current could be detected by the instrument. Therefore, the measurements of field emission properties were limited to the CoSi nanowires with different morphologies.

In addition to the field-emission properties, the electrical properties of the CoSi single-stem nanowires were also measured. The electrical measurements were carried out for single nanowires with various diameters. Pt electrodes were deposited at both ends of nanowires with a focus ion beam system. The resistivity reduces with decreasing radius of nanowires as illustrated in Figure 4. The resistivities and diameters of the nanowires are $287,181,100,27 \mu \Omega \mathrm{cm}$ and $43,34,23,15 \mathrm{~nm}$, respectively. The increase in conductivity with the decreasing diameter of nanowires is rather intriguing. It is attributed to the enhanced surface conductivity since the surface-to-volume ratio increases with the decreasing diameter. A previous work 
showed that the resistivity of $\mathrm{Pt}_{2} \mathrm{Si} \mathrm{NWs}$ is about half the reported resistivity of thick $\mathrm{Pt}_{2} \mathrm{Si}$ films. Additional conduction paths through surface or interface states on NWs were suggested to lead to the decrease in resistivity. ${ }^{33}$

\section{Summary and Conclusions}

A variety of cobalt silicide $\left(\mathrm{CoSi}, \mathrm{Co}_{2} \mathrm{Si}\right)$ nanostructures were grown by a chemical vapor transport method. Notably, novel three-dimensional (3D) CoSi nanowire networks and aloelike $\mathrm{Co}_{2} \mathrm{Si}$ nanostructures were grown under specific conditions in addition to nanowires. Both reaction temperature and vapor flow rate were found to critically influence the growth of nanostructures. The variation in Ar flow rates affected the densities and lengths of nanowires. On the other hand, the morphologies of the nanostructures and the diameter of nanowires were influenced by reaction temperature. Furthermore, the phases formed were determined by the Gibbs free energy changes in the reactions. Field-emission and electrical property measurements of CoSi nanowires show excellent performance with low turn-on field and good conductance. The CoSi nanowires are potentially useful in nanoelectronics devices.

Acknowledgment. The research was supported by the Republic of China National Science Council Grants NSC 96-2221-E-007-169-MY3 and NSC97-2120-M-007-003.

Note added after ASAP Publication. An earlier version of this paper was posted ASAP to the web on September 22, 2009, with an incorrect journal name in ref $10 \mathrm{a}$. The reference has been corrected in this new version posted October 7, 2009.

Supporting Information Available: Additional information (PDF). This material is available free of charge via the Internet at http://pubs.acs.org.

\section{References}

(1) Law, M.; Goldberger, J.; Yang, P. Annu. Rev. Mater. Res. 2004, 34, 83.

(2) Lu, W.; Lieber, C. M. Nat. Mater. 2007, 6, 841.

(3) Xia, Y.; Yang, P.; Sun, Y.; Wu, Y.; Mayers, B.; Gates, B.; Yin, Y.; Kim, F.; Yan, H. Adv. Mater. 2003, 15, 353.

(4) Chen, L. J. J. Mater. Chem. 2007, 17, 4639.

(5) He, J. H.; Hsin, C. L.; Liu, J.; Chen, L. J.; Wang, Z. L. Adv. Mater. 2007, 19, 781.

(6) Wang, Z. L. Adv. Mater. 2003, 15, 432.
(7) Huang, C. T.; Hsin, C. L.; Huang, K. W.; Lee, C. Y.; Yeh, P. H.; Chen, U. S.; Chen, L. J. Appl. Phv. Lett 2007, 91, 093133.

(8) Tian, B.; Zheng, X.; Kempa, T. J.; Fang, Y.; Yu, N.; Yu, G.; Huang, J.; Lieber, C. M. Nature 2007, 449, 885.

(9) Qin, Y.; Wang, X.; Wang, Z. L. Nature 2008, 451, 809.

(10) (a) Chen, L. J. JOM 2005, 57 (9), 24. (b) Lu, K. C.; Wu, W. W.; Wu, H. W.; Tanner, C. M.; Chang, J. P.; Chen, L. J.; Tu, K. N. Nano Lett. 2007, 7, 2389.

(11) Zhang, S. L.; Ostling, M. Crit. Rev. Solid State Mater. Sci. 2003, $28,1$.

(12) Wu, Y.; Xiang, J.; Yang, C.; Lu, W.; Lieber, C. M. Nature 2004, 430,61 .

(13) Zhang, S. L.; Smith, U. J.Vac. Sci. Technol., A 2004, 22, 1361.

(14) Lee, J. D.; Jin, S. H.; Shim, B. C.; Park, B. G. IEEE Electron. Device Lett. 2001, 22, 173.

(15) Chueh, Y. L.; Ko, M. T.; Chou, L. J.; Chen, L. J.; Wu, C. S.; Chen, C. D. Nano Lett. 2006, 6, 1637.

(16) Xiang, B.; Wang, Q. X.; Wang, Z.; Zhang, X. Z.; Liu, L. Q.; Xu, J.; Yu, D. P. Appl. Phvs. Lett. 2005, 86, 243103.

(17) Kim, C. J.; Kang, K.; Woo, Y. S.; Ryu, K. G.; Moon, H.; Kim, J. M.; Zang, D. S.; Jo, M. H. Adv. Mater. 2007, 19, 3637.

(18) Lee, C. Y.; Lu, M. P.; Liao, K. F.; Wu, W. W.; Chen, L. J. Appl. Phvs. Lett. 2008, 93, 113109.

(19) Lee, C. Y.; Lu, M. P.; Liao, K. F.; Lee, W. F.; Huang, C. T.; Chen, S. Y.; Chen, L. J. J. Phvs. Chem.C 2009, 113, 2286.

(20) Kang, K.; Kim, S.; Kim, C. J.; Jo, M. H. Nano Lett. 2008, 8, 431.

(21) Hsu, H. C.; Wu, W. W.; Hsu, H. F.; Chen, L. J. Nano Lett. 2007, 7, 885.

(22) Schmitt, A. L.; Zhu, L.; Schmeiaer, D.; Himpsel, F. J.; Jin, S. J. Phvs. Chem. B 2006, 37, 18142.

(23) Schmitt, A. L.; Bierman, M. J.; Schmeisser, D.; Himpsel, F. J.; Jin, S. Nano Lett. 2006, 6, 1617.

(24) Schmitt, A. L.; Higgins, J. M.; Jin, S. Nano. Lett. 2008, 8, 810.

(25) Ouyang, L.; Thrall, E. S.; Deshmukn, M. M.; Park, H. Adv. Mater. 2006, $18,1437$.

(26) Seo, K.; Varadwaj, K. S. K.; Mohanty, P.; Lee, S.; Jo, Y.; Jung, M. H.; Kim, J.; Kim, B. Nano Lett. 2007, 7, 1240.

(27) Szczech, J. R.; Schmitt, A. L.; Bierman, M. J.; Jin, S. Chem. Mater. 2007, 19, 3238

(28) Song, Y.; Schmitt, A. L.; Jin, S. Nano Lett. 2007, 7, 965.

(29) Varadwaj, K. S. K.; Seo, K.; In, J.; Mohanty, P.; Park, J.; Kim, B. J. Am. Chem. Soc. 2007, 129, 8594.

(30) Seo, K.; Varadwaj, K. S. K.; Cha, D.; In, J.; Kim, J.; Park, J.; Kim, B. J.Phvs. Chem.C 2007, 111, 9072.

(31) Seo, K.; Lee, S.; Yoon, H.; In, J.; Varadwaj, K. S. K.; Jo, Y.; Jung, M. H.; Kim, J.; Kim, B. ACS Nano 2009, 3, 1145.

(32) Du, J.; Du, P.; Hao, P.; Huang, Y.; Ren, Z.; Han, G.; Weng, W.; Zhao, G. J. Phvs. Chem. C 2007, 111, 10814.

(33) Lim, D. K.; Kubo, O.; Shingaya, Y.; Nakayama, T.; Kim, Y. H.; Lee, J. Y.; Aono, M.; Lee, H.; Lee, D.; Kim, S. Appl. Phv. Lett 2008, 92, 203114. 